\title{
The REFLEX galaxy cluster survey
}

\section{Spectroscopic observations and optical atlas ${ }^{\star}, \star \star$}

\author{
L. Guzzo ${ }^{1,2}$, P. Schuecker ${ }^{2}$, H. Böhringer ${ }^{2}$, C. A. Collins ${ }^{3}$, A. Ortiz-Gil ${ }^{1,4}$, S. De Grandi ${ }^{1}$, A. C. Edge ${ }^{5}$, \\ D. M. Neumann ${ }^{6}$, S. Schindler ${ }^{7}$, C. Altucci ${ }^{1}$, and P. A. Shaver ${ }^{8}$ \\ 1 INAF - Osservatorio Astronomico di Brera, via Bianchi 46, 23807 Merate (LC), Italy \\ e-mail: luigi.guzzo@brera.inaf.it \\ 2 Max-Planck-Institut für extraterrestrische Physik, Giessenbachstraße 1, 85740 Garching, Germany \\ 3 Astrophysics Research Institute, Liverpool John Moores University, Twelve Quays House, Egerton Wharf, \\ Birkenhead CH41 1LD, UK \\ 4 Observatori Astronomic - Universitat de Valencia, Edificio de Institutos de Investigacion, Aptdo. Correos 22085, 46071 Valencia, \\ Spain \\ 5 Physics Department, University of Durham, South Road, Durham DH1 3LE, UK \\ ${ }^{6}$ CEA Saclay, Service d'Astrophysique, Gif-sur-Yvette, France \\ 7 Institute for Astro- and Particle Physics, Universität Innsbruck, 6020 Innsbruck, Austria \\ 8 European Southern Observatory, 85748 Garching, Germany
}

Received 21 August 2008 / Accepted 16 December 2008

\section{ABSTRACT}

\begin{abstract}
We present the final data from the spectroscopic survey of the ROSAT-ESO Flux-Limited X-ray (REFLEX) catalog of galaxy clusters. The REFLEX survey covers 4.24 steradians (34\% of the entire sky) below a declination of $\delta=+2.5^{0}$ and at high Galactic latitude $\left(|b|>20^{\circ}\right)$. The REFLEX catalog includes 447 entries with a median redshift of 0.08 and is better than $90 \%$ complete to a limiting flux $f_{\mathrm{X}}=3 \times 10^{-12} \mathrm{erg} \mathrm{s}^{-1} \mathrm{~cm}^{-2}$ (0.1 to $\left.2.4 \mathrm{keV}\right)$, representing the largest statistically homogeneous sample of clusters drawn from the ROSAT All-Sky Survey (RASS) to date. Here we describe the details of the spectroscopic observations carried out at the ESO $1.5 \mathrm{~m}, 2.2 \mathrm{~m}$, and $3.6 \mathrm{~m}$ telescopes, as well as the data reduction and redshift measurement techniques. The spectra typically cover the wavelength range 3600-7500 $\AA$ at a two-pixel resolution of $\sim 14 \AA$, and the measured redshifts have a total rms error of $\sim 100 \mathrm{~km} \mathrm{~s}^{-1}$. In total we present 1406 new galaxy redshifts in 192 clusters, most of which previously did not have any redshift measured. Finally, the luminosity/redshift distributions of the cluster sample and a comparison to the no-evolution expectations from the cluster X-ray luminosity function are presented.
\end{abstract}

Key words. surveys - galaxies: clusters: general - galaxies: distances and redshifts

\section{Introduction}

Clusters of galaxies represent the largest collapsed objects in the hierarchy of cosmic structures, stemming from the growth of fluctuations lying on the high-density tail of the matter density field (Kaiser 1986). As such, their number density and evolution are strongly dependent on the normalization of the power spectrum and the value of the density parameter $\Omega_{\mathrm{M}}$ (e.g. Borgani \& Guzzo 2001; Rosati et al. 2002). In addition, the physics involved in "illuminating" clusters and making them visible is in principle easier to understand than the various complex processes connected to the formation and evolution of stars in galaxies (although a drawback can be that their typical dynamical time is long, comparable to the Hubble time). In particular in the X-ray band, where clusters can be defined and recognised as single objects (not just as a mere collection of galaxies),

\footnotetext{
* Based on data collected at the European Southern Observatory, La Silla, Chile

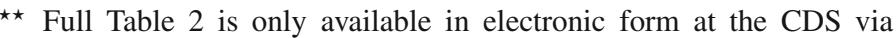
anonymous ftp to cdsarc.u-strasbg. fr $(130.79 .128 .5)$ or via http://cdsweb.u-strasbg.fr/cgi-bin/qcat?]/A+A/499/357, or htpp://www. brera.inaf.it/REFLEX
}

observable quantities like $\mathrm{X}$-ray luminosity $L_{\mathrm{X}}$ and temperature $T_{\mathrm{X}}$ show scaling relations with the total mass (and thus to the mass of the dark-matter halo, e.g. Evrard et al. 1996; Allen et al. 2001; Reiprich \& Böhringer 2002; Ettori et al. 2004). A full comprehension of these scaling relations requires more ingredients than the simple conversion of gravitational potential energy into heat during the growth of fluctuations (Kaiser 1986; Helsdon \& Ponman 2000; Finoguenov et al. 2001; Borgani et al. 2004). Nevertheless, these relations allow us to use clusters to test the mass function and the mass power spectrum, respectively via the observed cluster X-ray luminosity function (XLF) and clustering, (e.g. Böhringer et al. 2002; Pierpaoli et al. 2003; Schuecker et al. 2003a).

In addition to providing a fairly direct connection of observed quantities to model (mass-specific) predictions, X-ray based cluster surveys have further crucial advantages over optically-selected catalogs: first, X-ray emission is proportional to the gas density squared, and thus is more concentrated and less sensitive to projection effects than the simple galaxy density profile. Secondly, the selection function of an X-ray cluster survey is essentially that of a flux-limited sample, and thus fairly easy to reconstruct. This is a crucial feature when the goal is to 
use these samples for cosmological measurements that necessarily involve a precise knowledge of the sampled volume, as it is the case when computing first or second moments of the density field.

The advent of the ROSAT All-Sky Survey (RASS, Voges et al. 1999) at the beginning of the 1990's, opened up for the first time the possibility to construct X-ray cluster samples over wide areas of the sky. Optical identification of these clusters was eased by the good match between the flux limit of the RASS $\left(\sim 10^{-12} \mathrm{erg} \mathrm{s}^{-1} \mathrm{~cm}^{-2}\right.$ for extended sources) and the depth of the only wide-area optical imaging available at the time, i.e. the Palomar and in particular the southern UK-Schmidt sky surveys. These early X-ray samples included surveys like Hydra (Pierre et al. 1994), SGP (Romer et al. 1994; Cruddace et al. 2002, 2003), XBACS (Ebeling et al. 1996), BCS (Ebeling et al. 1998, 2000), RASS-BS (De Grandi et al. 1999), NORAS (Böhringer et al. 2000), NEP (Henry et al. 2001; Gioia et al. 2003). Some of these early studies concentrated on X-ray detections of opticallyselected clusters, i.e. typically systems previously identified optically by Abell (1958) and Abell et al. (1989), as notably the XBACS catalog or the surveys of Burns and collaborators (Burns et al. 1996; Ledlow et al. 1999). However, some others, as the SGP, BCS and RASS-BS surveys, were initial steps towards the goal of constructing a complete, X-ray selected statistical sample covering the whole sky, or at least the Southern hemisphere where deeper panoramic imaging was provided by the digitization of the ESO-SRC III-aJ $\left(b_{\mathrm{J}}\right)$ plates (through e.g. the Edinburgh-based COSMOS catalog, McGillivray \& Stobie 1984, or the APM survey, Maddox et al. 1990).

This goal has been achieved with the completion of the REFLEX (ROSAT-ESO Flux Limited X-ray) cluster survey, whose optical identification and spectroscopic survey are described here. REFLEX combines the X-ray data from the RASS and ESO follow-up optical observations to construct a complete flux-limited sample of 447 clusters with flux limit $f_{\mathrm{x}} \geq$ $3 \times 10^{-12} \mathrm{erg} \mathrm{s}^{-1} \mathrm{~cm}^{-2}$ (in the ROSAT energy band, $0.1-2.4 \mathrm{keV}$ ). It covers the Southern sky up to $\delta=+2.5^{\circ}$, excluding the band of the Milky Way $\left(\left|b_{\text {II }}\right| \leq 20^{\circ}\right)$ to avoid high NH column densities and crowding by stars. For the same reason, the regions of the Magellanic clouds are also excised from the survey (see Table 1 in Böhringer et al. 2001a, Paper I hereafter), totaling an overall area of $13924 \mathrm{deg}^{2}$ or 4.24 sr. The overall sky distribution of REFLEX clusters is shown in Fig. 1.

REFLEX provides the largest statistically complete X-rayselected cluster sample to date. The volume of Universe it probes is bigger than that covered by any present galaxy redshift survey except for the Sloan Digital Sky Survey, which goes to slightly larger depth but covers about half the sky area of REFLEX. We note that the RASS still remains today the only all-sky X-ray survey performed with an imaging X-ray telescope. We also note that the potential of the RASS for cluster research has not been fully exploited yet. There are two ongoing efforts in this direction. The REFLEX-2 survey is extending REFLEX to a fainter flux limit of $f_{\mathrm{x}}=1.8 \times 10^{-12} \mathrm{erg} \mathrm{s}^{-1} \mathrm{~cm}^{-2}$. This sample will contain more than 400 new clusters, part of which have been already observed spectroscopically during the ESO Key Programme described in this paper. Complementarily, the MACS survey (Ebeling et al. 2001, 2007) aims specifically at identifying all luminous X-ray clusters at $z>0.3$ still hiding in the RASS, probing an even bigger volume of the Universe.

The overall goal of REFLEX has been to map a large volume of the Universe using clusters, such that the survey could be used both to measure large-scale structure and as a controlled source for studying the physical properties of clusters. These requirements imposed a high standard to the whole X-ray source selection and identification process, which is described in detail in Böhringer et al. (2004, Paper II hereafter). In this paper, we present the data from the spectroscopic survey conducted with ESO telescopes to identify and measure the redshifts of REFLEX clusters. In particular, we report all relevant information on individual galaxy redshift data. We also provide (in electronic form), finding charts and optical/X-ray overlays of the clusters. These allow a first qualitative inspection of their main morphological properties (as e.g. their concentration or the presence of a dominant $\mathrm{cD}$ galaxy), which we hope will stimulate further quantitative work on this sample.

The complete REFLEX survey has been used over the last few years to measure fundamental cosmological quantities in the "local" Universe. These include, among others:

- the cluster X-ray luminosity function (Böhringer et al. 2002), and from this the mean abundance of clusters;

- the two-point correlation function of the cluster distribution (Collins et al. 2000);

- the power spectrum of the cluster distribution (Schuecker et al. 2001, 2002);

- the values of the cosmic mean density of matter $\Omega_{\mathrm{M}}$ and the power spectrum normalization $\sigma_{8}$, via the combination of the above observables (Schuecker et al. 2003a);

- the Gaussianity of the cluster distribution, as described by Minkowski functionals (Kersher et al. 2001);

- the value of the equation of state parameter of dark energy $w$ (Schuecker et al. 2003b);

- the relation between cluster velocity dispersions (measurable for a sub-sample of 170 clusters) and X-ray luminosity (Ortiz-Gil et al. 2004);

- the cluster-galaxy correlation function (Sanchez et al. 2005);

- the influence of scaling relation uncertainties on the estimate of cosmological parameters (Stanek et al. 2006).

One further general aspect is that through these measurements REFLEX provides the currently most robust local $(\langle z\rangle \sim 0.05)$ reference frame to which surveys of distant clusters can be safely compared in search of evolution (e.g. Borgani et al. 2001; Henry 2003; Stanford et al. 2006). Finally, the REFLEX catalog has provided the basis for statistically complete studies of the thermodynamical properties of the intra-cluster medium and the corresponding scaling relations. This is the case of the "REXCESS" XMM large survey recently completed (Boehringer et al. 2007).

The paper is organized as follows: in Sect. 2 we provide a quick overview of the selection and identification strategy of the REFLEX survey; in Sect. 3 we present the spectroscopic observations and discuss the observations, data reduction and redshift measurement technique; in Sect. 4 we present the spectroscopic catalog and the related finding charts and optical overlays; in Sect. 5 we discuss some properties of the redshift and luminosity distributions of REFLEX cluster; finally, in Sect. 6 we conclude and summarize the content of the paper. We adopt a "concordance" cosmological model, with $H_{\mathrm{o}}=70 \mathrm{~km} \mathrm{~s}^{-1} \mathrm{Mpc}^{-1}$, $\Omega_{\mathrm{M}}=0.3, \Omega_{\Lambda}=0.7$, and - unless specified - quote all X-ray fluxes and luminosities in the ROSAT [0.1-2.4] keV band.

\section{REFLEX identification strategy: overview}

We summarize here, for completeness, the main stages that led to the construction of the cluster candidate sample for REFLEX. A more comprehensive description can be found in Papers I and II.

The X-ray data for all sources detected in the RASS at declinations smaller than $2.5^{\circ}$ were analysed using the "Growth 
Table 1. Complete log of the spectroscopic observations.

\begin{tabular}{|c|c|c|c|c|c|c|c|}
\hline Date/Nights & & Tel. & Spectrograph & $\overline{\mathrm{CCD}}$ & $\begin{array}{l}\text { Grism/ } \\
\text { Grating }\end{array}$ & $\begin{array}{l}\text { Disp. } \\
\left(\AA \mathrm{mm}^{-1}\right)\end{array}$ & Detector model \\
\hline 1992, 27-31 May & (4) & $3.6 \mathrm{~m}$ & EFOSC-1 & $\# 16$ & B300 & 230 & Tek $512 \times 51230 \mu \mathrm{px}$ \\
\hline 1992, 1-5 Jun. & (5) & $1.5 \mathrm{~m}$ & $\mathrm{~B} \& \mathrm{C}$ & \#27 & \#21 & 130 & RCA $512 \times 51230 \mu \mathrm{px}$ \\
\hline 1992, 21-24 Nov. & (3) & $2.2 \mathrm{~m}$ & EFOSC-2 & \#19 & $\# 1$ & 442 & Thomson $1024 \times 102419 \mu \mathrm{px}$ \\
\hline 1992, 26-29 Nov. & (3) & $3.6 \mathrm{~m}$ & EFOSC-1 & \#26 & B300 & 230 & Tek $512 \times 51227 \mu \mathrm{px}$ \\
\hline 1993, 16-20 Apr. & (4) & $3.6 \mathrm{~m}$ & EFOSC-1 & \#26 & B300 & 230 & \\
\hline 1993, 13-19 Sep. & (6) & $1.5 \mathrm{~m}$ & $\mathrm{~B} \& \mathrm{C}$ & \#24 & \#27 & 114 & Ford $2048 \times 204815 \mu \mathrm{px}$ \\
\hline 1993, 14-17 Dec. & (3) & $3.6 \mathrm{~m}$ & EFOSC-1 & \#26 & B300 & 230 & \\
\hline 1994, 10-13 Mar. & (3) & $1.5 \mathrm{~m}$ & $\mathrm{~B} \& \mathrm{C}$ & \#24 & \#23 & 129 & \\
\hline 1994, 13-16 Mar. & (3) & $2.2 \mathrm{~m}$ & EFOSC-2 & \#19 & $\# 1$ & 442 & \\
\hline 1994, 6-11 Мay & (5) & $2.2 \mathrm{~m}$ & EFOSC-2 & \#19 & $\# 1$ & 442 & \\
\hline 1994, 12-15 May & (3) & $1.5 \mathrm{~m}$ & $\mathrm{~B} \& \mathrm{C}$ & \#24 & \#27 & 114 & \\
\hline 1994, 9-12 Sep. & (3) & $3.6 \mathrm{~m}$ & EFOSC-1 & \#26 & B300 & 230 & \\
\hline 1994, 6-9 Dec. & (3) & $3.6 \mathrm{~m}$ & EFOSC-1 & \#26 & B300 & 230 & \\
\hline 1994, 31 Dec. - 1995, 4 Jan. & (4) & $2.2 \mathrm{~m}$ & EFOSC-2 & \#19 & \#6 & 137 & \\
\hline 1995, 1-7 May & (6) & $2.2 \mathrm{~m}$ & EFOSC-2 & \#19 & \#6 & 137 & \\
\hline 1995, 25 Sep. - 1 Oct. & (6) & $2.2 \mathrm{~m}$ & EFOSC-2 & \#19 & \#6 & 137 & \\
\hline 1995, 20-23 Dec. & (3) & $3.6 \mathrm{~m}$ & EFOSC-1 & \#26 & B300 & 230 & \\
\hline 1995, 17-20 Dec. & (3) & $1.5 \mathrm{~m}$ & $\mathrm{~B} \& \mathrm{C}$ & \#39 & \#23 & 129 & Loral/Lesser $2048 \times 204815 \mu \mathrm{px}$ \\
\hline 1996, 7-10 Sep. & (3) & $3.6 \mathrm{~m}$ & EFOSC-1 & \#26 & B300 & 230 & \\
\hline 1996, 10-13 Sep. & (3) & $1.5 \mathrm{~m}$ & $\mathrm{~B} \& \mathrm{C}$ & \#24 & \#23 & 129 & \\
\hline 1997, 5-7 Feb. & (2) & $1.5 \mathrm{~m}$ & $\mathrm{~B} \& \mathrm{C}$ & \#39 & \#23 & 129 & \\
\hline 1997, 8-11 Feb. & (3) & $2.2 \mathrm{~m}$ & EFOSC-2 & \#40 & \#6 & 136 & Loral/Lesser $2048 \times 204815 \mu \mathrm{px}$ \\
\hline 1997, 1-2 Jun. & (1) & $3.6 \mathrm{~m}$ & EFOSC-1 & \#26 & B300 & 230 & \\
\hline 1997, 2-6 Jun. & (4) & $1.5 \mathrm{~m}$ & $\mathrm{~B} \& \mathrm{C}$ & \#39 & \#23 & 129 & \\
\hline 1997, 29 Sep. - 2 Oct. & (3) & $3.6 \mathrm{~m}$ & EFOSC-1 & \#26 & B300 & 230 & \\
\hline 1998, 30 Jan. - 1 Feb. & (1) & $3.6 \mathrm{~m}$ & EFOSC-2 & \#40 & \#11 & 136 & \\
\hline 1998, 17-20 Sep. & (3) & $1.5 \mathrm{~m}$ & $\mathrm{~B} \& \mathrm{C}$ & \#39 & \#23 & 129 & \\
\hline 1998, 20-22 Sep. & (2) & $3.6 \mathrm{~m}$ & EFOSC-2 & \#40 & \#11 & 136 & \\
\hline 1999, 17-20 May & (3) & $3.6 \mathrm{~m}$ & EFOSC-2 & \#40 & \#11 & 136 & \\
\hline
\end{tabular}

\section{REFLEX Cluster Survey}

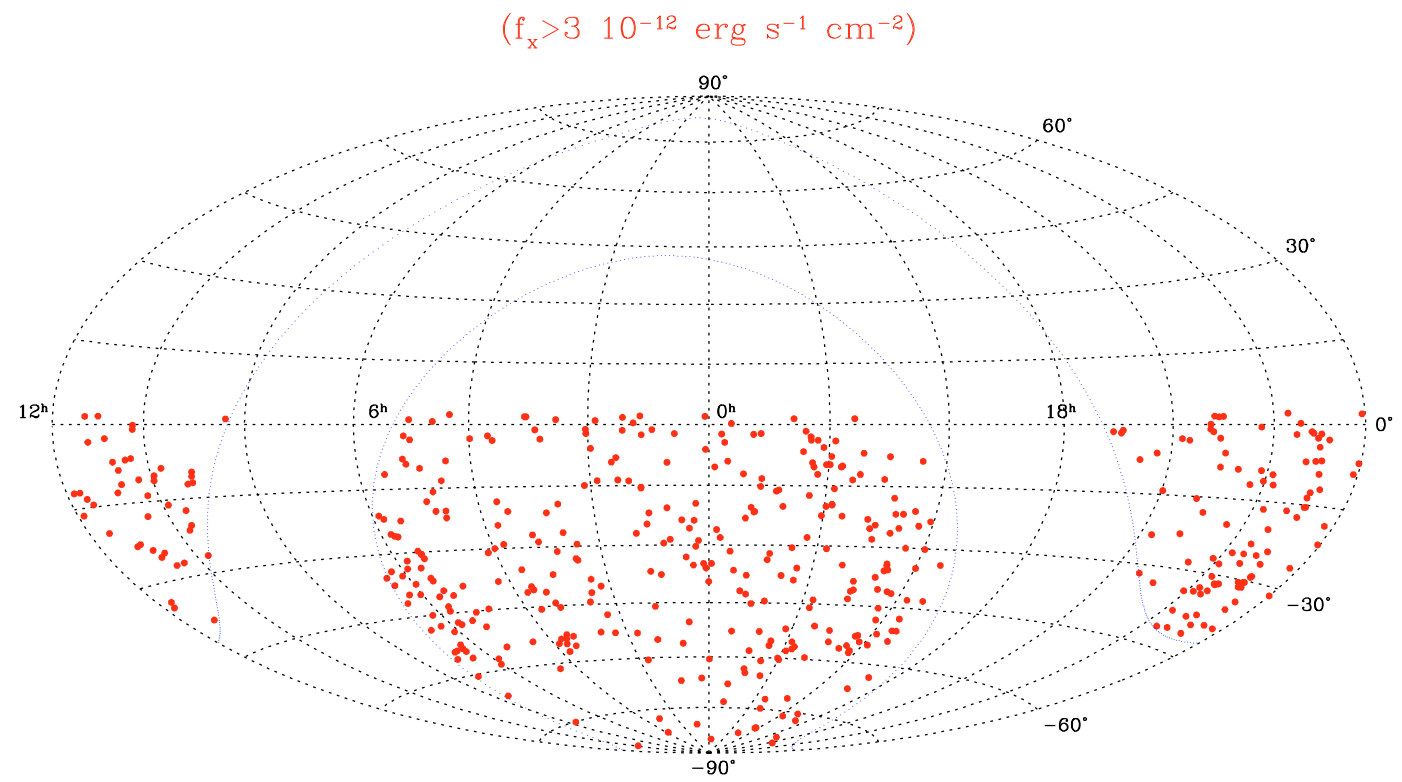

Fig. 1. The distribution on the sky of the 447 clusters composing the REFLEX sample with $f_{\mathrm{x}}>3 \times 10^{-12} \mathrm{erg} \mathrm{s}^{-1} \mathrm{~cm}^{-2}$. Note that the apparently blank area around $\alpha \sim 1^{\mathrm{h}}, \sim 5^{\mathrm{h}}, \delta \sim-70^{\circ}$ has been excised from the survey, corresponding to the Magellanic Clouds.

Curve Analysis" (GCA) method (Böhringer et al. 2000), thus re-measuring their flux and geometrical properties. The results are used to produce a flux-limited sample of RASS sources with $f_{\mathrm{x}} \geq 3 \times 10^{-12} \mathrm{erg} \mathrm{s}^{-1} \mathrm{~cm}^{-2}$. This redetermination of the fluxes has been shown to be crucial for extended RASS sources, as are the majority of REFLEX clusters (Ebeling et al. 1996; De Grandi et al. 1997; Böhringer et al. 2000). Cluster candidates were then found correlating all sources with galaxy density enhancements in the COSMOS optical data base, obtained from digital scans of the UK Schmidt survey plates at the 
Royal Observatory Edinburgh (MacGillivray \& Stobie 1984; Heydon-Dumbleton et al. 1989), with a density threshold low enough as to guarantee our desired final completeness of better than $90 \%$. This meant accepting a contamination of $\sim 30 \%$ by non-cluster sources spuriously associated with fluctuations in the galaxy background counts. The procedure ensures that the selection effects introduced by the optical identification process are minimized and negligible for our purpose (see also the statistics given in Paper I). Further tests provide support that a figure comfortably larger than $90 \%$ also describes the overall detection completeness of the flux-limited cluster sample in the survey area.

The resulting candidate list was then carefully checked against the available X-ray/optical information and with literature data, to eliminate obvious contaminants prior to the deeper optical follow-up observation program at La Silla. The adopted scheme was very conservative, again accepting a larger contamination (to be cleaned afterwards by the follow-up observations) to guarantee the highest possible completeness in the final sample (see Paper I for details).

The follow-up optical observations of REFLEX clusters were started at ESO in May 1992. With an "ESO Key Programme" status, the survey obtained an overall allocation of 90 nights, distributed among the ESO $3.6 \mathrm{~m}, 2.2 \mathrm{~m}$ and $1.5 \mathrm{~m}$ telescopes. A few additional nights were further obtained at the end of the project to partly compensate for the time lost due to bad weather. The complete observing log of the survey is presented in Table 1.

The goal of these observations was twofold: a) obtain a definitive identification of ambiguous candidates; b) obtain a measurement of the mean cluster redshift. First, a number of candidate clusters required direct CCD imaging and/or spectroscopy to be safely included in the sample. For example, candidates characterised by a poor appearance on the Sky Survey IIIa-J plates, with no dominant central galaxy or featuring a seemingly point-like X-ray emission had to pass further investigation. In this case, either the object at the X-ray peak was studied spectroscopically, or a short CCD image plus a spectrum of the 2-3 objects nearest to the X-ray peak were taken. This operation was preferentially scheduled at the two smaller telescopes (1.5 $\mathrm{m}$ and $2.2 \mathrm{~m}$, see below). In this way, a few AGN's were discovered. When the overall information available (e.g. X-ray hardness ratio, source shape) was consistent with the AGN dominating the emission, the corresponding candidate was rejected from the main list. This is described in full detail in Paper II, where also a list of the more uncertain or ambiguous cases in the REFLEX catalog is presented and discussed thoroughly.

For the bonafide clusters, the final goal of the optical observations was then to secure a reliable redshift. The observing strategy was designed as a compromise between the desire of having several redshifts per cluster, coping with the multiplexing limits of the available instrumentation, and the large number of clusters to be measured. Previous experience on the similar Edinburgh/Milano Survey of EDCC clusters (Collins et al. 1995), had shown the importance of not relying on just one or two galaxies to measure the cluster redshift, especially for clusters without a dominant $\mathrm{cD}$ galaxy. However, the additional information provided by the detection and localisation of X-rays makes the issues of projection - that make multiple member redshifts vital for optical samples - much less severe here ${ }^{1}$.

\footnotetext{
${ }^{1}$ In fact, the data from the REFLEX survey itself show exactly this: $\mathrm{X}$-ray emission provides an extremely good guidance towards targeting galaxies which have a high probability to be cluster members (see
}

EFOSC1 in MOS mode was a perfect instrument for getting quick redshift measurements for 10-15 galaxies at once, but only for systems that could reasonably fit within the small field of view of the instrument (5.2 arcmin side in imaging with the Tektronics CCD \#26, but less than 3 arcmin for spectroscopy in MOS mode, due to hardware/software limitations in the making of the MOS masks). This feature made this combination useful only for clusters above $z \sim 0.1$, i.e. where at least the core region could be accommodated within the available area (a core radius of $0.1 \mathrm{~h}^{-1} \mathrm{Mpc}$ is seen under an angle of $1.3 \mathrm{arcmin}$ at such redshift, in the adopted cosmology).

The other important aspect of this instrumental set-up is that in several cases, after removal of background/foreground objects one is still left with $8-10$ galaxy redshifts within the cluster, by which a first estimate of the cluster velocity dispersion can be attempted. This has been done, complementing the data described here with literature redshifts, for a sub-sample of 170 REFLEX clusters, allowing us to study the scaling relation between cluster velocity dispersion and X-ray luminosity (Ortiz-Gil et al. 2004).

At lower redshifts, doing efficient multi-object spectroscopy work on cluster fields would have required a MOS spectrograph with a larger field of view, i.e. 20-30 arcmin diameter. One possible choice could have been the formerly available ESO fibre spectrograph Optopus (Avila et al. 1989), but its efficiency in terms of numbers of targets observable per night was too low for covering the several hundred clusters we had in our sample. We found the best solution was to split the work between the $1.5 \mathrm{~m}$ and $2.2 \mathrm{~m}$ telescopes. Clearly, this required accepting some compromise in our initial goal of having multiple redshifts for each cluster. As discussed in Paper II, about half of the cluster redshifts are measured with 5 or more member galaxies, but 42 of them featuring only one galaxy redshift. Most of these cases come from the literature, and the available telescope budget did not allow for a re-determination of these values. For most of these cases, however, the reliability of these single redshift as estimators of the mean systemic redshift is high, as they refer to the brightest cluster galaxy at the centre of X-ray emission. Indeed, as mentioned above, the coupling of the galaxy positions with the X-ray contours is of strong help in indicating which galaxies have the highest probability to be cluster members.

During 8 years of work, we have observed spectroscopically a total of about 500 cluster candidates, collecting over 3200 galaxy spectra. In this paper, we present the spectroscopic data belonging to the current, published REFLEX sample with $f_{\mathrm{x}}>3 \times 10^{-12} \mathrm{erg} \mathrm{s}^{-1} \mathrm{~cm}^{-2}$, which include $\sim 1500$ spectra. The remaining spectra belong to clusters extending to fainter fluxes, which will form part of a deeper REFLEX-2 sample reaching to $f_{\mathrm{x}}=1.8 \times 10^{-12} \operatorname{erg~s}^{-1} \mathrm{~cm}^{-2}$.

\section{Spectroscopy}

\subsection{Observations}

As detailed in Table 1, all spectroscopic observations were performed at the ESO La Silla observatory, using the $3.6 \mathrm{~m}, 2.2 \mathrm{~m}$ and $1.5 \mathrm{~m}$ telescopes. In the following, we describe the instrumental set-ups and main data properties for each of them.

also Crawford et al. 1999). This allows the cluster mean redshift to be constrained with fewer objects than for a "blind" survey of opticallyselected clusters, as the EDCC. 


\subsubsection{EFOSC1/2@3.6 m observations}

About $80 \%$ (in terms of number of spectra) of all the REFLEX survey observations were carried out using the $3.6 \mathrm{~m}$ telescope with the ESO Faint Object Spectrograph and Camera (EFOSC) in its two incarnations - EFOSC1 and EFOSC2. The EFOSC instruments are high-efficiency transmission spectrographs, with multi-object spectroscopic capability (MOS) and fast switching to imaging mode. This latter feature allows very accurate slit positioning on faint objects. At the time of completing this paper (2008), EFOSC-2 is still actively used at the $3.6 \mathrm{~m}$ telescope.

Table 1 shows how during the development of the survey different detectors were installed on the EFOSCs, following the evolution of CCD's technology. The EFOSCs were mostly used in MOS mode, which entailed producing aluminium masks of the cluster fields, on which slitlets of 5-30 arcsec length were carved following a direct image taken with the same instrument. The masks were then inserted into free positions in the aperture wheel of the spectrograph. The width of the slits was always of 2-arcsec, the same width used for single-slit observations. Depending on the available CCD-grism combination, which varied during the survey, we worked at dispersions ranging between 130 and $230 \AA / \mathrm{mm}$, usually aiming at a wavelength coverage between $3600 \AA$ and $7500 \AA$. Most of the $3.6 \mathrm{~m}$ observations were performed using EFOSC-1 with the B300 grism at $230 \AA / \mathrm{mm}$ and a Tektronics $512 \times 512$ chip, yielding a resolution of $6.9 \AA$ per pixel. This corresponds to a spectral resolution (as measured on a purely instrumentally broadened line) of $\sim 2$ pixels $F W H M$, providing radial velocity errors well below $100 \mathrm{~km} \mathrm{~s}^{-1}$ for good $S / N$ ratio spectra obtained from two consecutive exposures of 10-15 mn each (see Sect. 3.3.3 for details). On average each mask contained 15-20 slitlets, over the available $5.2 \times 5.2 \operatorname{arcmin}^{2}$ field of view. Standard calibration observations were collected, as discussed in more detail in Sect. 3.2. A number of single-slit observations were also carried out at the $3.6 \mathrm{~m}$ telescope, with a similar set-up, especially near the end of the survey, targeting some of the most distant clusters in the sample.

\subsubsection{EFOSC2@2.2 m and B\&C@1.5 m observations}

A fraction of the spectroscopic observations were carried out in single-slit mode using either EFOSC-2 at the $2.2 \mathrm{~m}$ telescope, (before this instrument was moved to the $3.6 \mathrm{~m}$ telescope in January 1998), or the 1.5 m telescope, equipped with a classical Boller \& Chivens spectrograph coupled with an RCA (1992) or Ford (1993 onwards) CCD. The latter had very poor response in the blue range, i.e. below $4500 \AA$, where some of the most interesting absorption lines (e.g. Calcium $H$ and $K$, $\mathrm{G}$-band) fall for galaxies at low redshift. Thus, the $1.5 \mathrm{~m}$ telescope was essentially reserved to observe the brightest members (up to $m_{\mathrm{B}} \sim 17.5$ ) in the more nearby clusters of the sample, and played a minor role in the overall redshift survey. The spectral setup was similar to that adopted for the EFOSC spectrographs (grating \#21, $172 \AA \mathrm{mm}^{-1}$, blaze angle $\theta=6^{\circ} 54^{\prime}$ ).

\subsection{Data reduction}

The data were reduced using the MIDAS (for data prior to 1995) and IRAF spectroscopic packages, using either custombuilt programs (for MIDAS) or - for the bulk of the data - the IRAF specific set of procedures (TWODSPEC/APEXTRACT). The set of operations performed on the available long-slit or MOS spectroscopic CCD frames followed the usual standard procedures, and was essentially the same in both environments. For two observing runs, we repeated the full data reduction using both packages and a direct comparison of the calibrated spectra showed differences well below our typical radial velocity errors $\left(<30 \mathrm{~km} \mathrm{~s}^{-1}\right)$. In the following, we shall limit ourselves, for simplicity, to the IRAF version of the reduction pipeline which in the end was used for most of the spectra, describing its various phases.

- CCD frame inspection, quality check and standardization. These operations included in particular checks for: (a) Possible systematic time dependences of the average bias; (b) possible shifts of the sky lines during the observing run. Sky line positions were also checked after wavelength calibration (see below); (c) similarly, possible shifts of $\mathrm{He} / \mathrm{Ar} / \mathrm{Ne}$ comparison lines at different times during the observing run. All available science and calibration frames were then trimmed to a common size, to eliminate spurious extra borders and overscan regions.

- Bias and flat-field corrections. Multiple sets of bias frames were regularly collected during each observing night and combined through a $3 \sigma$-clipping algorithm (ZEROCOMBINE), to produce a single, two-dimensional bias frame for that night. In general, the bias frames from the $1.5 \mathrm{~m}$, from the $2.2 \mathrm{~m}$, and from the $3.6 \mathrm{~m}$ telescope showed twodimensional structures at the $<0.5$ percent level (rms) which are removed by this procedure. To flat-field our spectra only dome flats were typically observed in day time during each run, given that we were not aiming at precise spectrophotometry. Median flats were constructed for each run using IMCOMBINE. The number of effectively used flat field exposures for each Single-Slit spectroscopic run ranged between 7 and 40. In practice, we observed virtually no effect when flat-fielding data from the $1.5 \mathrm{~m}$ and the $3.6 \mathrm{~m}$ observations, while this operation was crucial for most of the data collected at the $2.2 \mathrm{~m}$. Only in the very last $2.2 \mathrm{~m}+\mathrm{EFOSC} 2$ run (February 1997) a new CCD (\#40) was installed, eliminating this problem (EFOSC2 was then moved later in that year to the $3.6 \mathrm{~m}$ telescope, where we then performed most of the subsequent observations). We concluded that there was no gain in flat-fielding the MOS observations collected at the $3.6 \mathrm{~m}$ telescope with EFOSC1 (CCD \#26) and EFOSC2 (CCD \#40). Finally the two (or more) science exposures available for every spectroscopic observation were averaged together with IMCOMBINE, after appropriate scaling and weighting by the the exposure time. This removed very effectively most of the cosmic ray events.

- Science and comparison spectra extraction. Twodimensional spectra corresponding to each slit were then extracted following possible curvature of the spectrum. These were then reduced to 1-D sky-subtracted spectra using proper sky background regions in the slit. All operations were performed within the APALL/APEXTRACT environment of IRAF. Corresponding 1D calibration spectra were also extracted at exactly the same positions from all the available lamp exposures associated with the target frame. These were typically two $\mathrm{He}-\mathrm{Ar}$ arc frames, observed before and after the science exposure. At the $3.6 \mathrm{~m}$ telescope, our direct tests for instrument flexures using 5 strong $\mathrm{He}$ lines at 7 extreme telescope positions showed an rms shift $<0.12$ pixels, corresponding to $0.744 \AA$, i.e. $45 \mathrm{~km} \mathrm{~s}^{-1}$. We concluded that EFOSC flexures over the time of one typical $3.6 \mathrm{~m}$ observation $(\sim 30 \mathrm{mn})$ were negligible, and 

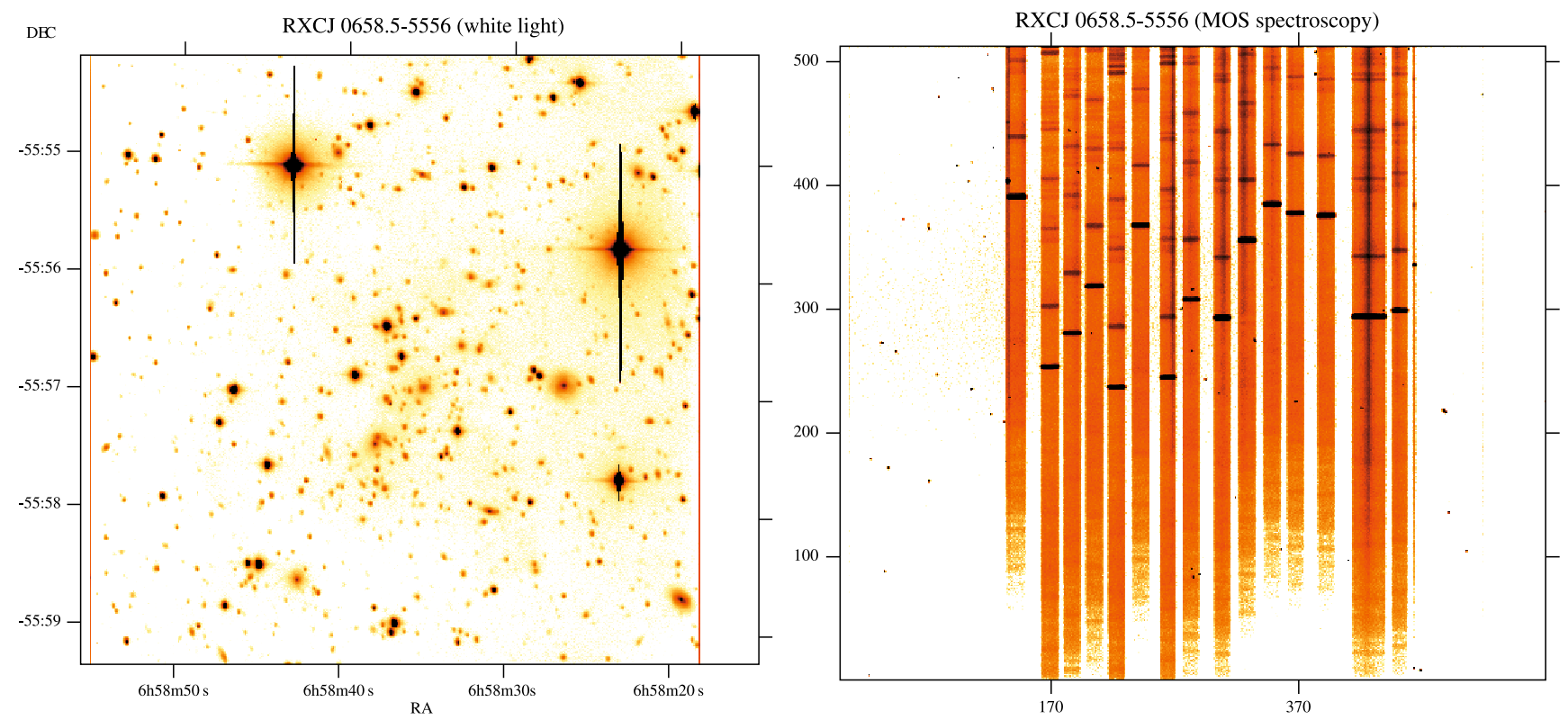

Fig. 2. Example of a direct image and spectral lay-out for a cluster observed in MOS mode with EFOSC-1 at the $3.6 \mathrm{~m}$ telescope. Left: direct image in white light of RXCJ0658.5-5556, a luminous REFLEX cluster at $z=0.2965$ (see also Fig. 4). Right: the resulting MOS frame, showing the set of two-dimensional spectra corresponding to each target galaxy in the mask. The dispersion runs along the vertical direction. Each strip shows the sky spectrum (dark horizontal lines) together with the fainter galaxy spectrum.

subsequently used only one calibration lamp. This was not the case at the $1.5 \mathrm{~m}$ and $2.2 \mathrm{~m}$ telescopes, as shown by measurements by the ESO staff. For these data, we used both arcs observed before and after the science exposures to compute a time-averaged set of reference lines, as feasible within the IRAF procedures.

- Wavelength calibration. The whole operation was automatized through the available IRAF procedures (IDENTIFY/REIDENTIFY). In general, an accurate pixelto-wavelength transformation was determined for the first spectrum of either a full night of long-slit spectroscopy or a single MOS exposure. The residuals were directly inspected and discrepant arc line identifications eliminated. The procedure was iterated until a satisfactory rms was reached. The relation was then applied to the science spectrum using DISPCOR. Typical rms wavelength calibration errors ranged between $\sim 0.3 \AA$ (for the majority of spectra, e.g. those taken at the $3.6 \mathrm{~m}$ telescope), to $\sim 1 \AA$ for lower resolution spectra as those obtained at the $2.2 \mathrm{~m}$ telescope with grism \#1. All other spectra in a night-series of long-slit observations were then calibrated by using the first solution as a guess, using REIDENTIFY. For MOS spectra, however, where large shifts in the zero point between adjacent spectra are normal (see Fig. 2), the position of a bright Helium line was used to provide an approximate zero-point shift for each spectrum. This was done through a custom-developed script, and provided the first-guess to calibrate all spectra in a MOS frame with the usual procedure. The quality and consistency of the final calibration was counter-checked a posteriori by measuring the position of the three brightest sky lines ([OI]

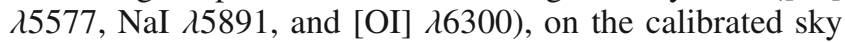
spectrum of each extracted science slit. This allowed us to spot and correct a few pathological cases.

- Final cleaning and heliocentric corrections. Before feeding the 1D wavelength-calibrated spectra to the crosscorrelation analysis, a number of quality checks and final corrections were performed. These include cleaning of bright sky line residuals (via both an automatic cleaning routine plus visual inspection), computation of heliocentric corrections using the RVCORRECT package (typically smaller than $30 \mathrm{~km} \mathrm{~s}^{-1}$ ). Before actually feeding the spectra to the crosscorrelation routine, emission lines were removed automatically, as only absorption-line templates were used for the measurement. Emission-line redshifts were estimated separately, using the specific routine EMSAO.

\subsection{Redshift measurements}

\subsubsection{Cross-correlation technique}

Galaxy redshifts were estimated from the 1D calibrated spectra, using the classical cross-correlation technique described in detail by Tonry \& Davis (1979). This is implemented within the IRAF environment through the package RVSAO (Kurtz \& Mink 1998). The basis of the technique is the cross-correlation of the observed galaxy spectrum with a model or template spectrum. This is performed by taking the Fast Fourier Transform of the two spectra, multiplying them together and then transforming back the result to get the Cross-Correlation Function (CCF), whose highest peak is related to the radial velocity difference between the two spectra. Before actually starting this machinery, the two spectra are rebinned into logarithmic bins, so that the relative redshift becomes a linear shift. Then, a number of operations are performed on the spectra, in order to improve the signal-to-noise of the final cross-correlation function. These include continuum subtraction, apodizing and bandpass filtering. All these operations are performed inside the XCSAO routine of RVSAO. We tested several combinations of the command parameters to find the most appropriate set for our spectra. For example, the values for the low- and high-frequency cut-offs of the bandpass filter are specific for the kind of data being used, and optimal values were chosen after experimenting, as to maximize the significance of the CCF. Filtering is important in order to eliminate both the low frequency spurious components left by 

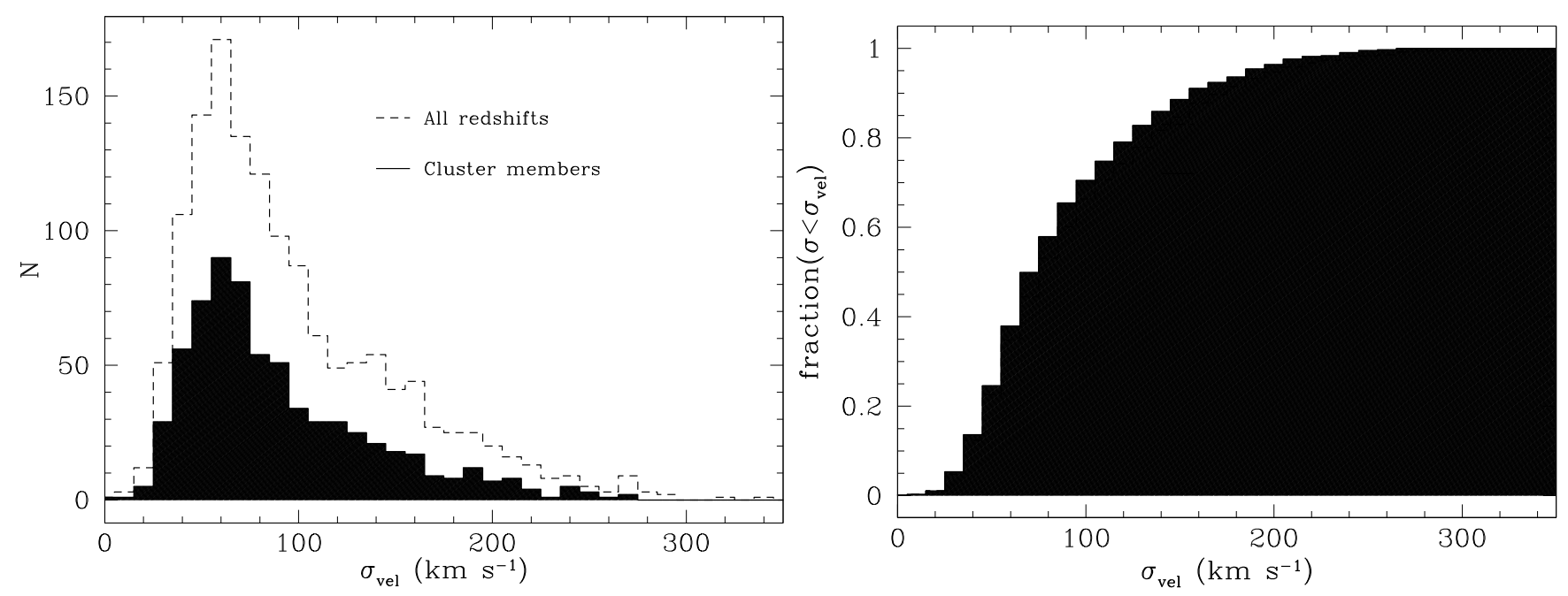

Fig. 3. Left: differential distribution of the errors on galaxy radial velocities in the REFLEX survey, as estimated by RVSAO. The filled histogram corresponds to considering only cluster member galaxies (i.e. those that were actually used to compute mean cluster redshifts). Collins et al. (1995) showed that total redshift errors amount to typically between 1 and 2 times the internal error estimate by RVSAO, depending inversely on the SNR of the spectrum. Accounting for this, and considering that all spectra around or to the left of the peak of the distribution have very good SNR, we conclude that the typical total error on single galaxy redshifts remains below $100 \mathrm{~km} \mathrm{~s}^{-1}$. This assures that the dominant source of uncertainty in the mean cluster redshift will be the intrinsic velocity dispersion of the cluster and not a big uncertainty on single galaxy measurements.

the subtracted continuum, and the high frequency binning noise. Also, we tested that the redshift estimate was quite insensitive to the exact binwidth (corresponding to 2048 or 4096 bins) chosen in the rebinning. The peak of the CCF was fit by a quadratic polynomial, determining the wavelength shift from its position, and providing an estimate of the uncertainty from its width.

\subsubsection{Template spectra}

At the core of the cross-correlation technique is the comparison of the object spectrum with a model spectrum of known radial velocity and ideally infinite $S / N$ ratio, the template. The key point of the technique lies in the remarkable similarity in the basic features among galaxy spectra, although the relative intensity of absorption lines can vary quite significantly, in particular when different morphological types are considered. In practice, to cover the range of spectral properties a number of different templates is used for each object and the one producing the highest cross-correlation peak is then taken to be the best model, at the resulting redshift, of the galaxy spectrum being measured.

For measuring the spectra of the REFLEX survey, we benefited of the accurate set of templates constructed by Ettori et al. (1995, EGT hereafter), to which we refer for all details on their properties and construction. This set of templates has a number of useful properties. One advantage is that it includes separate stellar and galaxy spectra together with composite spectra. Another important feature is the accurate knowledge of the template zero points, calibrated in EGT using a set of highresolution "primary" stellar templates.

This template library includes 17 spectra: two highresolution HD stars with accurately known radial velocity, 3 high $S / N$ galaxies observed with EFOSC in a previous project (Collins et al. 1995), and combined stellar and galaxy spectra built by EGT.

\subsubsection{Redshift errors}

The major advantage of the cross-correlation technique (Tonry \& Davis 1979) is to make use of the complete redshift information contained in the whole spectrum, not just in the few major identifiable lines. This pushes the measurement errors well below that expected from the nominal spectral resolution used. Depending on the SNR of the spectrum, errors as small as $1 / 10$ of the nominal accuracy on one single-line measurement are achieved. The specific IRAF implementation RVSAO computes a confidence level $R$ of the chosen CCF peak as the ratio of the peak height to the rms background of the CCF. We empirically verified that estimates with $R<4$ have to be treated with caution, while larger values normally indicated a rather secure value. We also used the stability of the redshift value provided by the different templates as an extra figure of merit. Each galaxy spectrum was crosscorrelated against the 17 templates described above. The overall results for each template were directly inspected and spectra with 5 or more templates in agreement (within the redshift errors) and $R>4$ were passed as secure. Spectra that did not satisfy these criteria strictly, had in several cases between 2 and 4 templates in agreement. Visual inspection of these cases often supported the suggested redshift. The typical features of cluster early-type galaxy spectra, as in particular the $4000 \AA$ break, make the visual check of the suggested redshift fairly straightforward. Spectra were discarded if (1) there was no agreement between the templates; and (2) the visual inspection did not indicate a plausible redshift. Once a spectrum had been accepted as secure (visually or with $\geq 5$ templates), the template redshift with the highest $R$ parameter level was assigned to the galaxy. If several templates had the same confidence, then the one with the lowest returned internal error was used. For high signal-tonoise spectra, it was common to find all the templates agreeing to within a scatter of $\Delta v \simeq 50 \mathrm{~km} \mathrm{~s}^{-1}$. The distribution of the errors for the final redshifts is plotted in Fig. 3. According to these histograms, the median formal error on our galaxy redshifts is $\sim 60 \mathrm{~km} \mathrm{~s}^{-1}$, with $70 \%$ of them being better than $100 \mathrm{~km} \mathrm{~s}^{-1}$. A small fraction of the galaxies had emission lines in their spectra. These are indicated in the redshift catalog, together with the corresponding emission-line radial velocity. This is normally of lower accuracy than the global, cross-correlation based redshift which uses the information from the whole absorption spectrum, and has been used to compute the cluster redshift only when no 
Table 2. Sample page from the full catalog of REFLEX galaxy redshifts (full table available at the CDS and at http://www. brera.inaf.it/ REFLEX).

\begin{tabular}{|c|c|c|c|c|c|c|c|c|c|c|c|}
\hline $\begin{array}{c}\text { REFLEX } \\
\text { target }\end{array}$ & $\begin{array}{c}\text { RA } \\
\text { (hh:mm:ss) }\end{array}$ & $\begin{array}{c}\text { DEC } \\
\text { (dd:mm:ss) }\end{array}$ & Type & $\begin{array}{l}\text { Cluster } \\
\text { member }\end{array}$ & $\begin{array}{l}c z_{\mathrm{abs}} \\
\left(\mathrm{km} \mathrm{s}^{-1}\right)\end{array}$ & err & $\bar{R}$ & $\begin{array}{c}c z_{\mathrm{em}} \\
\left(\mathrm{km} \mathrm{s}^{-1}\right)\end{array}$ & Date & $\overline{\overline{\text { Tel }}}$ & Notes \\
\hline RXCJ0014.3-6604 & $0: 13: 56.88$ & $-66: 04: 14.5$ & galaxy & - & 82756 & 142 & 3.0 & & 01-Nov.-92 & $3.6 \mathrm{~m}$ & low SNR \\
\hline RXCJ0014.3-6604 & $0: 14: 00.24$ & $-66: 04: 59.9$ & galaxy & - & 132089 & 42 & 3.3 & 37260 & 01-Nov. -92 & $3.6 \mathrm{~m}$ & low SNR \\
\hline RXCJ0014.3-6604 & $0: 14: 01.27$ & $-66: 04: 39.4$ & galaxy & - & 184316 & 123 & 2.9 & & 01-Nov. -92 & $3.6 \mathrm{~m}$ & low SNR \\
\hline RXCJ0014.3-6604 & $0: 14: 04.94$ & $-66: 05: 38.0$ & galaxy & - & 36817 & 87 & 3.2 & & 01-Nov-92 & $3.6 \mathrm{~m}$ & low SNR \\
\hline RXCJ0014.3-6604 & $0: 14: 05.93$ & $-66: 05: 35.5$ & galaxy & + & 47946 & 90 & 3.6 & & 01-Nov.-92 & $3.6 \mathrm{~m}$ & low SNR \\
\hline RXCJ0014.3-6604 & $0: 14: 05.35$ & $-66: 04: 21.0$ & galaxy & + & 48051 & 74 & 6.9 & & 01-Nov. -92 & $3.6 \mathrm{~m}$ & - \\
\hline RXCJ0014.3-6604 & $0: 14: 11.66$ & $-66: 04: 41.9$ & $\mathrm{cD}$ gal. & + & 45963 & 59 & 8.9 & & 01-Nov.-92 & $3.6 \mathrm{~m}$ & - \\
\hline RXCJ0014.3-6604 & $0: 14: 15.53$ & $-66: 05: 38.8$ & galaxy & - & 25197 & 93 & 3.0 & & 01-Nov. -92 & $3.6 \mathrm{~m}$ & low SNR \\
\hline RXCJ0014.3-6604 & $0: 14: 16.20$ & $-66: 04: 21.7$ & galaxy & + & 47729 & 72 & 5.9 & & 01-Nov.-92 & $3.6 \mathrm{~m}$ & - \\
\hline RXCJ0014.3-6604 & $0: 14: 19.58$ & $-66: 04: 52.3$ & star & - & -251 & 43 & 8.4 & & 01-Nov.-92 & $3.6 \mathrm{~m}$ & - \\
\hline RXCJ0014.3-6604 & $0: 14: 22.08$ & $-66: 04: 57.7$ & galaxy & + & 48638 & 72 & 3.5 & & 01-Nov.-92 & $3.6 \mathrm{~m}$ & low SNR \\
\hline RXCJ0014.3-6604 & $0: 14: 26.95$ & $-66: 04: 17.1$ & galaxy & - & 74613 & 94 & 3.7 & & 01-Nov. -92 & $3.6 \mathrm{~m}$ & low SNR \\
\hline RXCJ0014.3-6604 & $0: 14: 28.92$ & $-66: 04: 44.1$ & star & - & 12 & 77 & 4.6 & & 01-Nov.-92 & $3.6 \mathrm{~m}$ & - \\
\hline RXCJ0017.5-3509 & $0: 17: 34.87$ & $-35: 11: 00.2$ & galaxy & + & 28071 & 100 & 11.1 & & 16-Sep.-93 & $1.5 \mathrm{~m}$ & - \\
\hline RXCJ0017.5-3509 & $0: 17: 31.87$ & $-35: 11: 54.6$ & galaxy & + & 29653 & 151 & 9.8 & & 16-Sep.-93 & $1.5 \mathrm{~m}$ & - \\
\hline RXCJ0027.3-5015 & $0: 27: 27.60$ & $-50: 14: 31.9$ & galaxy & - & 67964 & 127 & 3.6 & & 19-Sep.-93 & $1.5 \mathrm{~m}$ & low SNR \\
\hline RXCJ0027.3-5015 & $0: 27: 23.45$ & $-50: 14: 40.6$ & galaxy & - & 38130 & 220 & 4.7 & 37795 & 19-Sep.-93 & $1.5 \mathrm{~m}$ & low SNR \\
\hline RXCJ0027.3-5015 & 0:27:20.66 & $-50: 14: 46.3$ & galaxy & + & 43399 & 71 & 13.4 & & 19-Sep.-93 & $1.5 \mathrm{~m}$ & - \\
\hline RXCJ0042.1-2832 & $0: 41: 56.26$ & $-28: 30: 43.9$ & galaxy & - & 16878 & 267 & 3.3 & & 01-Nov.-92 & $2.2 \mathrm{~m}$ & - \\
\hline RXCJ0042.1-2832 & $0: 41: 58.99$ & $-28: 31: 08.0$ & galaxy & - & 15892 & 176 & 5.3 & & 01-Nov.-92 & $2.2 \mathrm{~m}$ & - \\
\hline RXCJ0042.1-2832 & $0: 42: 02.42$ & $-28: 31: 34.3$ & galaxy & - & 16492 & 232 & 4.3 & & 01-Nov.-92 & $2.2 \mathrm{~m}$ & - \\
\hline RXCJ0042.1-2832 & $0: 42: 08.90$ & $-28: 32: 08.5$ & galaxy & - & 16135 & 168 & 5.5 & & 01-Nov.-92 & $2.2 \mathrm{~m}$ & - \\
\hline RXCJ0042.1-2832 & $0: 42: 11.78$ & $-28: 32: 35.5$ & galaxy & - & 16253 & 154 & 5.5 & & 01-Nov.-92 & $2.2 \mathrm{~m}$ & - \\
\hline RXCJ0042.1-2832 & $0: 42: 14.86$ & $-28: 32: 55.7$ & galaxy & - & 15572 & 275 & 3.3 & & 01-Nov.-92 & $2.2 \mathrm{~m}$ & - \\
\hline RXCJ0042.1-2832 & $0: 42: 08.26$ & $-28: 32: 07.8$ & galaxy & + & 33305 & 75 & 9.0 & & 01-Nov.-92 & $2.2 \mathrm{~m}$ & - \\
\hline RXCJ0042.1-2832 & $0: 42: 08.90$ & $-28: 32: 08.5$ & cD gal. & + & 32296 & 86 & 10.1 & & 01-Nov. -92 & $2.2 \mathrm{~m}$ & - \\
\hline
\end{tabular}

absorption redshift was available. From our previous experience with the same instrumental set-up (Collins et al. 1995), we also know that the external error on the radial velocities (measured from repeated observations of the same galaxies) is between a factor of one and two larger than the XCSAO internally estimated value (depending on the SNR). This implies a median value for the total measurement errors of $\sim 100 \mathrm{~km} \mathrm{~s}^{-1}$.

\subsection{Galaxy astrometry}

Precise astrometric coordinates were assigned a posteriori to each observed spectrum, since they were available only approximately from the header of the spectroscopic frames. For singleslit observations this was in general straightforward, as (especially at the two smaller telescopes) these involved fairly bright galaxies. For MOS observations, on the other hand, it required a significant amount of work, as unfortunately no electronic information on the astrometric position of target galaxies on the MOS slits is saved along with the observations at the telescope. For this reason, we calibrated astrometrically all the direct CCD images available for each spectroscopic target field, using the USNO2 galaxy catalog and the Starlink's Graphical Astronomy and Image Analysis Tool (GAIA, http: //star-www . dur . ac. uk/pdraper/gaia/gaia.html). This was made possible by using the service white-light CCD images used to prepare the EFOSC slit masks, that were appropriately saved at the time of observations. For some fields, images in $B$ and $R$ bands were also available. Inevitably, the final match of the 1D spectra and redshift to their specific galaxy position on the sky was then performed by hand, using the astrometrically calibrated images and the HEDIT IRAF task to write RA and DEC in the spectrum FITS header.

\section{Catalog of galaxy redshifts and optical data base}

\subsection{Galaxy redshift catalog}

During our spectroscopic observing campaign, we collected new redshifts for 192 clusters which are included in the current REFLEX catalog. Additionally, a number of systems with X-ray fluxes fainter than the current REFLEX limit were also measured, together with candidates that were subsequently discarded as non-cluster sources. The full list of measured galaxy redshifts for clusters in the REFLEX sample is provided in electronic form only (see wWw. brera.inaf.it/REFLEX). Here we provide only an excerpt, which is displayed in Table 2. The columns give, respectively: (1) REFLEX name, as defined in Paper II; $(2,3)$ Coordinates J2000 of each target galaxy; (4) Simple spectral classification, to distinguish among stars, galaxies and clear AGN-like spectra. This classification is not meant to be exhaustive. Additionally, when clear from the available imaging, the spectroscopic measurement of the $\mathrm{cD}$ galaxy is explicitly noted; (5) Assignment as a cluster member (+) or interloper (-); (6) heliocentric redshift $c z$ in $\mathrm{km} \mathrm{s}^{-1}$, as measured from absorption lines through the cross-correlation procedure; (7) corresponding 

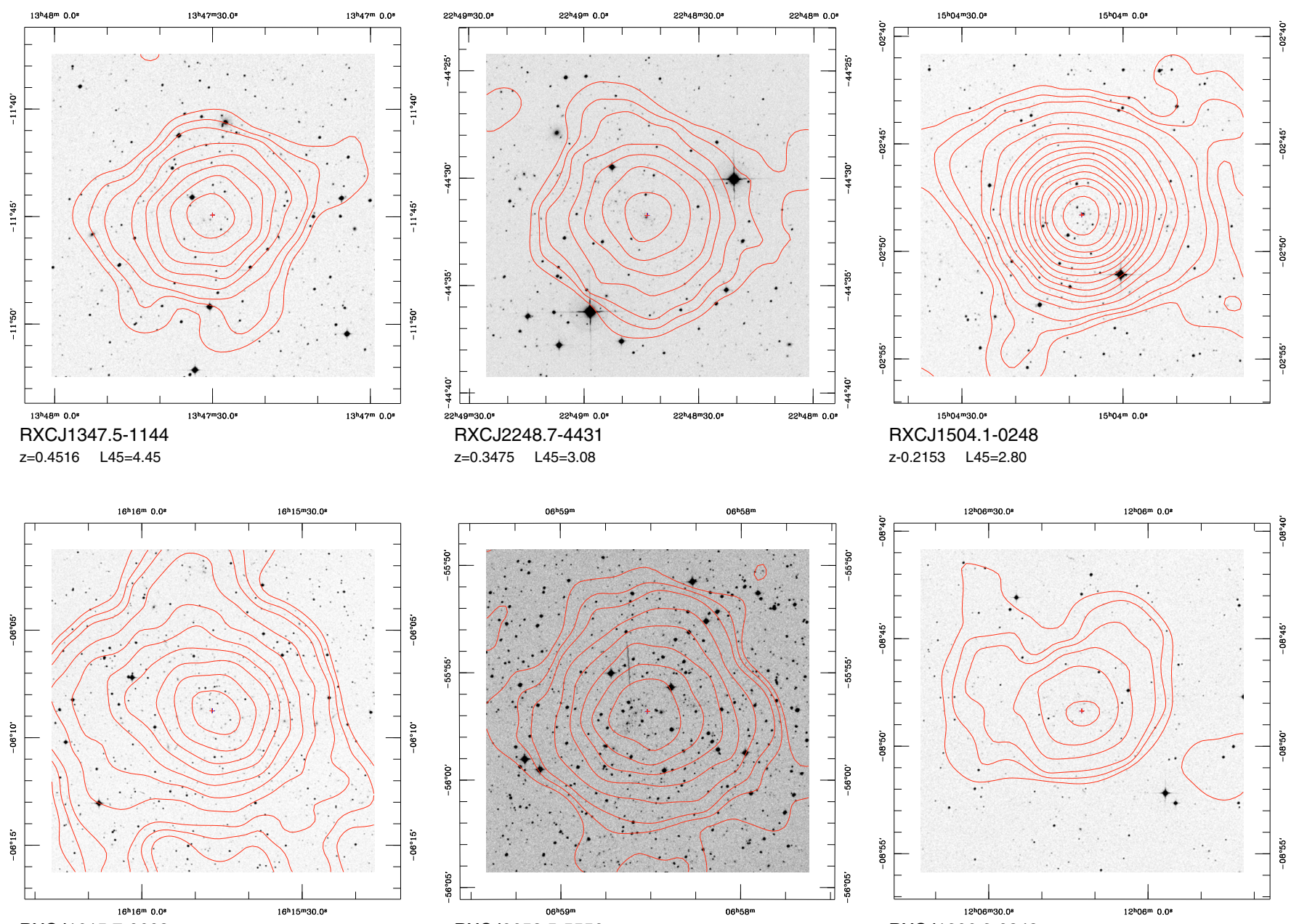

RXCJ1615.7-0608
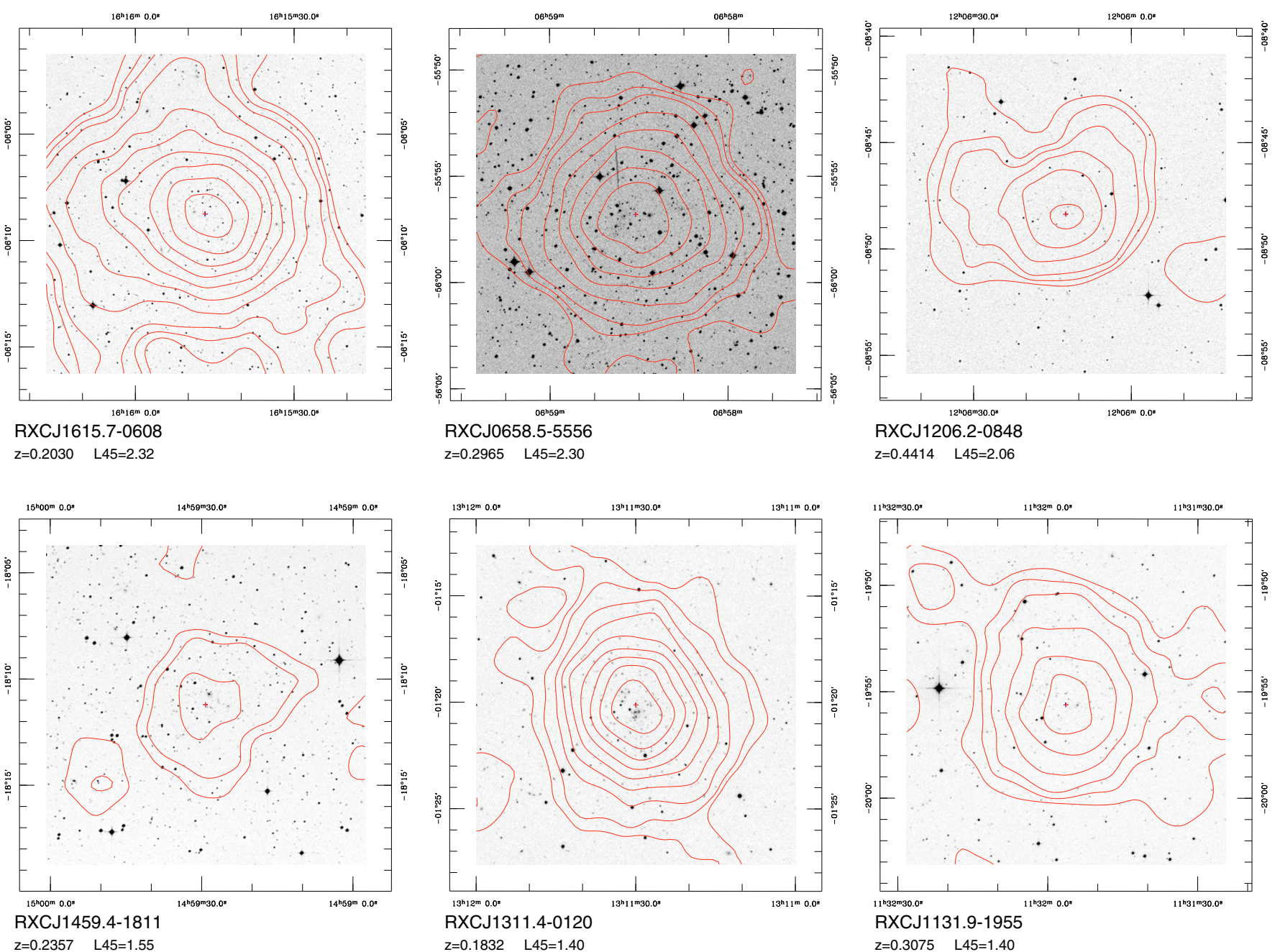

Fig. 4. Overlays of the X-ray emission in the [0.5-2.0] KeV band plotted onto the DSS2-RED images of clusters in the REFLEX surveys. The contours correspond to steps of $1 \sigma$ in the significance of the X-ray emission, defined as the rms fluctuation within a Gaussian window of 1-arcmindispersion of the ratio $S / \sqrt{B+S}$, where $S$ is the source signal and $B$ the mean value of the background. We show here the 9 most luminous systems in the survey. For each cluster, we also report its redshift and X-ray luminosity in units of $10^{45} \mathrm{erg} \mathrm{s}^{-1} \mathrm{~cm}^{-2}$ (as given in Paper II). The full set of overlays and finding charts is available at higher resolution from the survey web page. These clusters include objects already known from previously existing catalogs, as A2163 (RXCJ1615.7-0608), A1689 (RXCJ1311.4-0120) and A1300 (RXCJ1131.9-1955) from the Abell catalog (Abell 1958) or S1063 (RXJ2248.7-4431) and S0780 (RXCJ1459.4-1811) from its Supplementary list. Notable is also RXCJ0658.5-5556, which corresponds to the famous "Bullet Cluster" (Clowe et al. 2006) originally discovered by the Einstein observatory as 1ES 0657-558. 


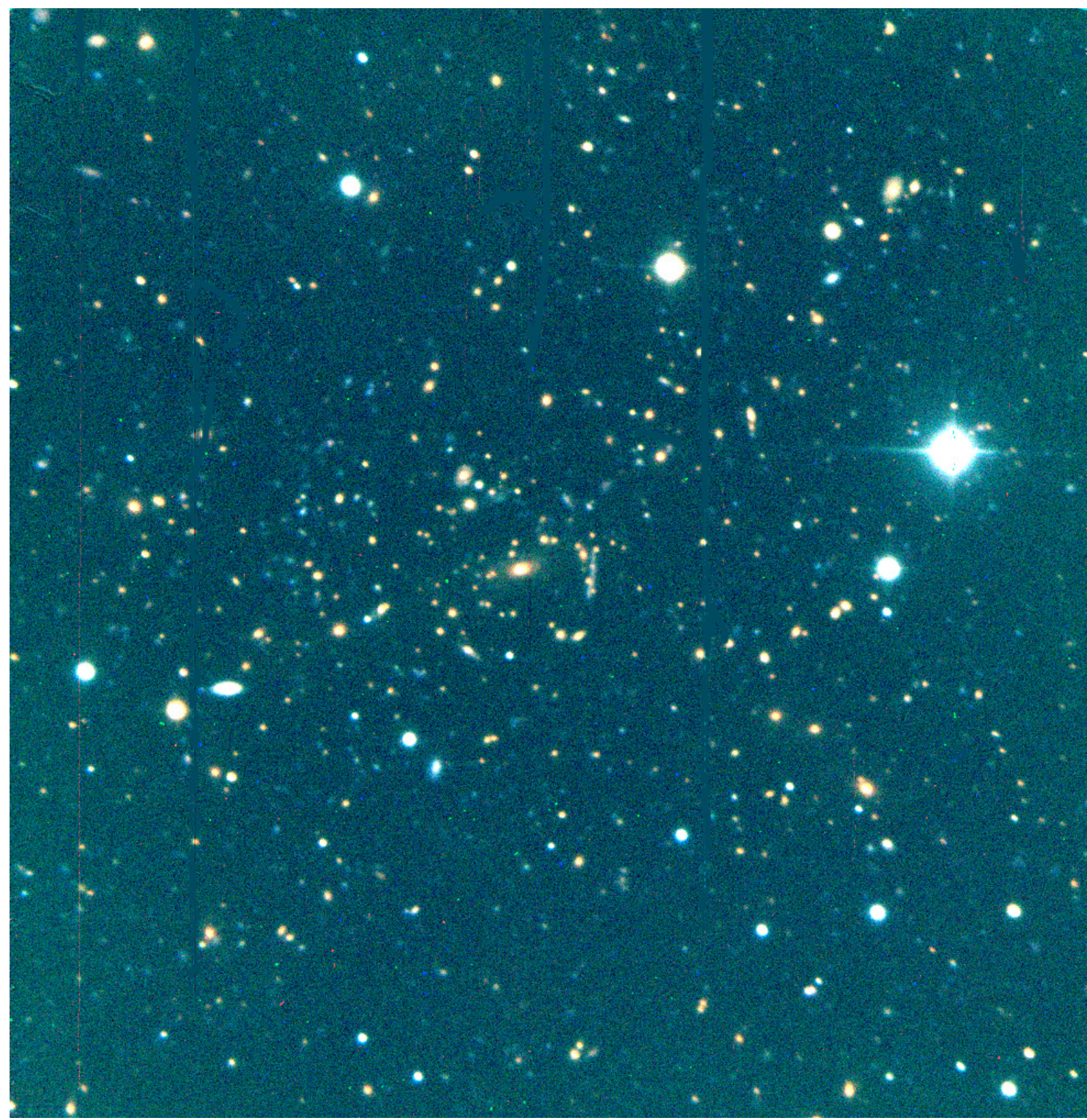

Fig. 5. Composite RGB image of RXCJ1206.2-0848, one of the most spectacular new clusters discovered by the REFLEX survey (the sixth most luminous shown in Fig. 4). This image has been built combining three short (10 mn) direct exposures in the Johnson $B, V$ and $R$ bands taken with EFOSC2 at the ESO $3.6 \mathrm{~m}$ telescope and is 5.5 arcmin on a side. Note the dominating $\mathrm{cD}$ with very extended yellowish halo, and the prominent blue gravitational lensing candidate arc just westward of it. Several other possible arclets are also visible.

error; (8) redshift confidence parameter $R$, giving the ratio between the height of the cross-correlation peak and the overall rms noise of the same function; (9) emission-line redshift, when available; $(10,11)$ Observation date and telescope; (12) When needed, notes on the spectrum quality or on possible problems in the reduction, astrometry or redshift quality.

\subsection{Finding charts and optical/X-ray atlas}

A complete optical/X-ray atlas of images for the REFLEX clusters, including finding charts for the spectroscopically measured galaxies is too big to be included in this paper. We have therefore set up a visual atlas of DSS finding charts and X-ray overlays, which is accessible through the survey web page (WWW . brera.inaf.it/REFLEX). The scientific content of the X-ray overlays and their construction are discussed in a separate paper (Böhringer et al., in preparation). The web page will also be used to present future upgrades of the REFLEX catalog, or new information on single clusters.

As a visual example of the most spectacular objects which are part of the catalog, we show here a printed version of the overlays for the nine most luminous REFLEX clusters (Fig. 4). Some of these are famous clusters already known before REFLEX, as detailed in the caption. Some others are new objects discovered by REFLEX. These include, for example RXCJ1347.4-1144 at $z=0.4516$, the most luminous X-ray cluster known to date (Schindler et al. 1995). Another spectacular example of these newly discovered systems is RXCJ1206.2-0848 at $z=0.4414$, for which we show in Fig. 5 an RGB composite of three CCD images in the $B, V$ and $R$ bands. 
We hope the easy-to-browse cluster imaging atlas will be useful for planning specific studies of cluster sub-classes, as e.g. cD clusters or the so-called "fossil" groups (of which REFLEX includes a remarkable sub-set).

\section{Luminosity and spatial distribution}

The new redshifts for galaxies in REFLEX clusters presented here have been used, together with a large bulk of existing data from the literature, to assign a systemic redshift to each cluster, as described in Paper II and to compute a velocity dispersion for a sub-set of 170 objects, as reported in Ortiz-Gil et al. (2004). In Paper II, we already presented a first discussion of the sample resulting from the redshift survey, mostly concentrating on the unambiguous identification of the redshift system related to the $\mathrm{X}$-ray source. We briefly summarize some of these aspects here, presenting some further details on the properties of the REFLEX cluster sample and its spatial distribution.

Figure 6 shows the redshift distribution of the 447 clusters included in the REFLEX catalog. As a consistency check, this is compared to the curve one obtains by integrating as a function of redshift the no-evolution X-ray luminosity function (XLF hereafter) measured from the sample itself (Böhringer et al. 2002). Figure 7, instead, plots the X-ray luminosity $L_{X}$ of the clusters as a function of redshifts. The plot shows how the REFLEX sample is able to include some of the most X-ray luminous clusters in the Universe, thanks to its large volume. It is evident how all very luminous systems with $L_{\mathrm{X}}>10^{45} \mathrm{erg} \mathrm{s}^{-1}$ are found above $z>0.15$. This is the consequence of these clusters being rare fluctuations lying on the exponential tail of the luminosity function: at any redshift, there is a maximum luminosity $L_{\mathrm{MAX}}$, above which the expected number of clusters (given by the integral of the luminosity function $\phi(L)$ above $L_{\text {MAX }}$ times the volume explored), drops below unity. Following Sandage et al. (1979), the value of $L_{\mathrm{MAX}}$ as a function of redshift is implicitly provided by the expression

$N\left(<z,>L_{\text {MAX }}\right)=1$,

i.e.

$$
\int_{0}^{V(z)} \mathrm{d} V \int_{L_{\mathrm{MAX}}}^{\infty} \mathrm{d} L \phi(L)=1 .
$$

The corresponding solution $L_{\mathrm{MAX}}(z)$, given the REFLEX XLF corresponds to the dashed curve in Fig. 7. The curve describes fairly well the upper envelope of the $L_{X}-z$ plot, with fluctuations around it produced by large-scale structures (where the mean density, and thus the normalization $\phi^{*}$ of the XLF fluctuates around the mean value used in the computation).

Finally, Fig. 8 provides an overview of the 3D distribution of REFLEX clusters, within $z<0.2$. One can easily notice the level of structure still existing on such very large scales, with a number of evident aggregations of clusters with sizes $\sim 100 \mathrm{~h}^{-1} \mathrm{Mpc}$.

\section{Summary}

The REFLEX survey consists of 447 galaxy clusters constituting the largest statistically complete (to better than 90\%) $\mathrm{X}$-ray flux-limited cluster survey to date. The spectroscopic follow-up of REFLEX was carried out as part of an ESO Key Programme using a combination of single slit and multi-object spectroscopy providing new redshifts for 1406 galaxies in these systems. Clusters were observed with either the Faint Object Spectrograph and Camera on the $2 \mathrm{~m}$ and $3.6 \mathrm{~m}$ ESO telescopes

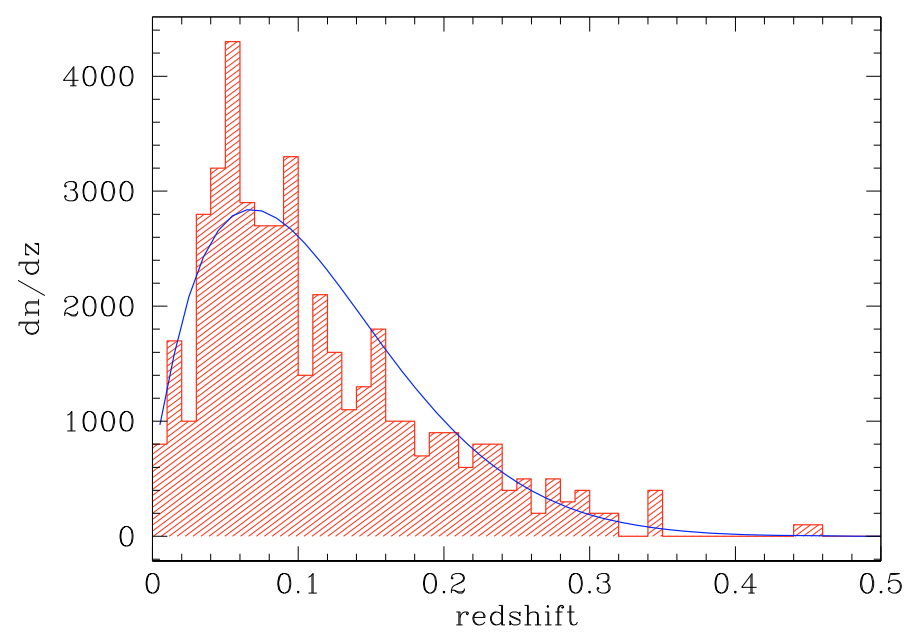

Fig. 6. Redshift distribution of the REFLEX clusters (histogram), compared to that expected from integration of the REFLEX X-ray Luminosity Function from Böhringer et al. (2002).

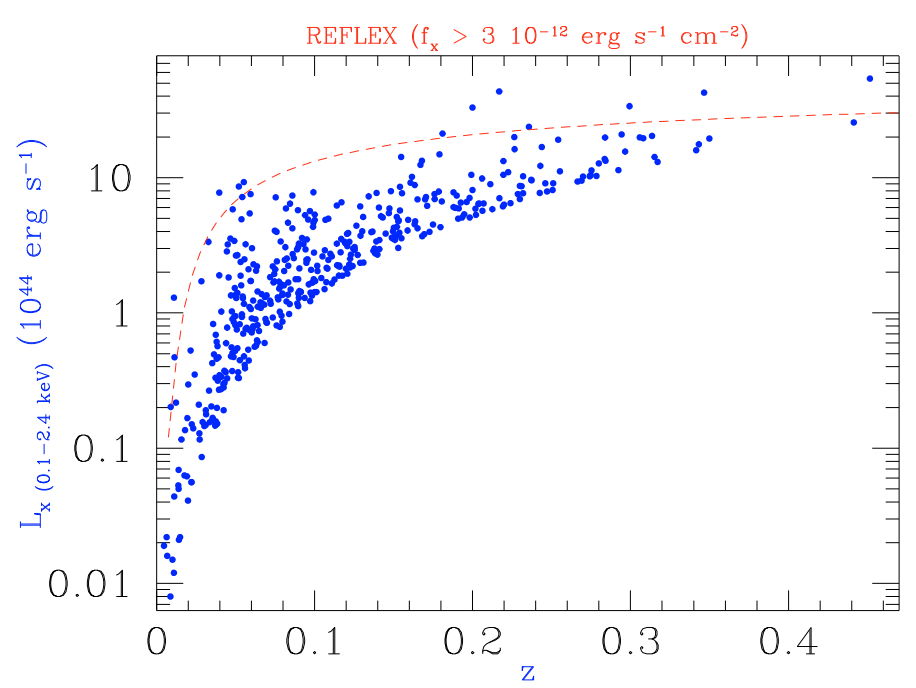

Fig. 7. X-ray luminosity versus redshift for the final REFLEX sample of galaxy clusters. The lower cut-off in $L_{\mathrm{X}}$ corresponds to the survey flux limit of $3 \times 10^{-12} \mathrm{erg} \mathrm{s}^{-1} \mathrm{~cm}^{-2}$. The upper dashed line gives instead the expected volume effect imposed by the luminosity function. It is computed as the luminosity value above which, at every redshift, less than 1 cluster is expected within the enclosed cosmological volume. This curve shows clearly how only with a volume as big as that of REFLEX one can adequately explore the bright end of the luminosity function.

or the Boller and Chivens spectrograph on the $1.5 \mathrm{~m}$. These combinations provide a spectral wavelength coverage of between $3600-8000 \AA$ and a two-pixel resolution of $\simeq 14 \AA$. Redshifts are measured mainly by cross-correlation with a range of template spectra.

Internal fitting errors and external comparisons indicate that galaxy redshifts are typically accurate to $100 \mathrm{~km} \mathrm{~s}^{-1}$, with errors as small as $50 \mathrm{~km} \mathrm{~s}^{-1}$ for the highest SNR spectra. We have produced optical/X-ray overlays for all clusters, together with 


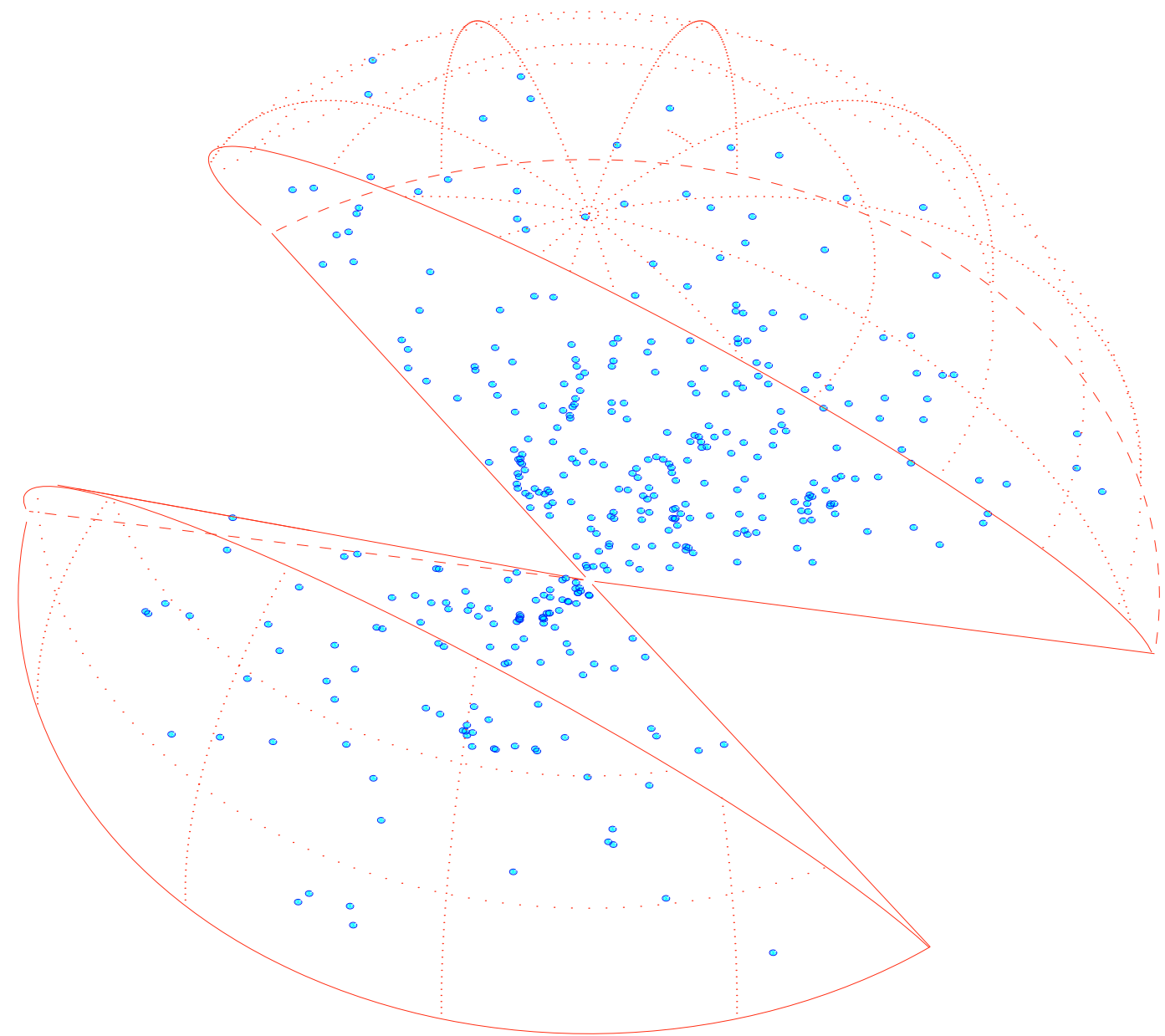

Fig. 8. The large-scale spatial distribution of REFLEX clusters within $600 \mathrm{~h}^{-1} \mathrm{Mpc}$. The South Galactic Pole is here placed on top to ease display. The missing wedge is the region occupied by the Galactic plane $\left( \pm 20^{\circ}\right)$.

finding charts indicating the spectroscopically observed galaxies. These are available at http://www.brera.inaf.it/ REFLEX, together with the complete table of all galaxy redshifts.

Acknowledgements. We would like to thank G. Chincarini, R. Cruddace, H. MacGillivray, T. Reiprich, K. Romer, W. Seitter, P. Vettolani, W. Voges and G. Zamorani for their contribution and support to various aspects of the REFLEX project. The help of U. Briel, R. Dümmler, H. Ebeling, G. Guerrero, D. Lazzati, E. Molinari, S. Molendi and D. Wake during some observing sessions is also gratefully acknowledged. We thank A. Mistò for the development and maintenance of the Brera database system, which has been vital to support parts of this work. The redshift survey of REFLEX clusters was performed in the framework of the ESO "Key Programmes". We would like to acknowledge the generous allocation of telescope time by ESO OPC. We thank the ESO-La Silla staff for invaluable support, and in particular F. Patat and M. Kurster for special assistance during observations at the $3.6 \mathrm{~m}$ telescope. We thank the referee, Harald Ebeling, for his comments that helped to improve the presentation of the paper. L.G. acknowledges financial support from MIUR (through grant PRIN 98 "Galaxy Formation and Evolution"), the Italian Space Agency (ASI) and the hospitality of the Excellence Cluster "Universe" in Garching (supported by DfG grant EXC 153), where this paper was completed. AOG has been supported by the Spanish MEC project AYA2003-08739-C02-01 (including FEDER) and by the Generalitat Valenciana ACyT project GRUPOS03/170.

This research has made use of the NASA/IPAC Extragalactic Database (NED) which is operated by the Jet Propulsion Laboratory, California Institute of Technology, under contract with the National Aeronautics and Space Administration. It has also made use of NASA's Astrophysics Data System.

This paper and the whole REFLEX project would not have been possible without the dedication of Peter Schuecker, who passed away in November 2006. He has been the driving force behind both the observations and the key scientific results obtained by the REFLEX survey. We all miss his theoretical knowledge, pure approach to science and unique humanity.

\section{References}

Abell, G. O. 1958, ApJS, 3, 211

Abell, G. O., Corwin, H. G., \& Olowin, R. P. 1989, ApJS, 70, 1

Allen, S. 2001, MNRAS, 328, 37

Avila, G., DOdorico, S., Tarenghi, M., \& Guzzo, L. 1989, The Messenger, 55, 62

Böhringer, H., Voges, W., Huchra, J. P., et al. 2000, ApJS, 129, 435

Böhringer, H., Schuecker, P., Guzzo, L., et al. 2001a, A\&A, 369, 826 (Paper I)

Böhringer, H., Schuecker, P., Komossa, S., et al. 2001b, in Mapping the Hidden Universe, ed. R. C. Kraan-Korteweg, P. A. Henning, \& H. Andernach, 93 [arXiv: astro-ph/0011461]

Böhringer, H., Collins, C. A., Schuecker, P., et al. 2002, ApJ, 566, 93

Böhringer, H., Schuecker, P., Guzzo, L., et al. 2004, A\&A, 425, 367 (Paper II)

Böhringer, H., Schuecker, P., Pratt, G. W., et al. 2007, A\&A, 469, 363

Borgani, S., \& Guzzo, L. 2001, Nature, 409, 39

Borgani, S., Rosati, P., Tozzi, P., et al. 2001, ApJ, 561, 13

Borgani, S., Murante, G., Springel, V., et al. 2004, MNRAS, 348, 1078

Burns, J. O., Ledlow, M. J., Loken, C., et al. 1996, ApJ, 467, L49

Clowe, D., Bradac, M., Gonzalez, A. H., et al. 2006, ApJ, 648, L109

Collins, C. A., Guzzo, L., Nichol, R. C., \& Lumsden, S. L. 1995, MNRAS, 274, 1071

Collins, C. A., Guzzo, L., Böhringer, H., et al. 2000, MNRAS, 319, 939

Crawford, C. S., Allen, S. W., Ebeling, H., Edge, A. C., \& Fabian, A. C. 1999, MNRAS, 306, 857

Cruddace, R., Voges, W., Böhringer, H., et al. 2002, ApJS, 140, 239

De Grandi, S., Molendi, S., Böhringer, H., \& Voges, W. 1997, ApJ, 486, 738

De Grandi, S., Böhringer, H., Guzzo, L., et al. 1999, ApJ, 514, 148

Ebeling, H., Voges, W., Böhringer, H., et al. 1996, MNRAS, 281, 799

Ebeling, H., Edge, A. C., Böhringer, H., et al. 1998, MNRAS, 301, 881

Ebeling, H., Edge, A. C., Allen, S. W., et al. 2000, MNRAS, 318, 333

Ettori, S., Guzzo, L., \& Tarenghi, M. 1995, MNRAS, 276, 689 (EGT)

Ettori, S., Tozzi, P., Borgani, S., \& Rosati, P. 2004, A\&A, 417, 13 
Evrard, G., Metzler, C. A., \& Navarro, J. F. 1996, ApJ, 469, 494

Gioia, I. M., Henry, J. P., Mullis, C. R. et al. 2003, ApJS, 149, 29

Finoguenov, A., Reiprich, T. H., \& Böhringer, H. 2001, A\&A, 368, 749

Guzzo, L., Böhringer, H., Schuecker, P., et al. 1999, The Messenger, 95, 27

Helsdon, S. F, \& Ponman, T. J. 2000, MNRAS, 319, 933

Henry, J. P. 2003, in Matter and Energy in Clusters of Galaxies, ed. S. Bowyer, \& C.-Y. Hwang, ASP Conf. Proc., 30, San Francisco, 5

Henry, J. P., Gioia, I. M., Mullis, C. R., et al. 2001, ApJ, 553, L109

Heydon-Dumbleton, N., Collins, C. A., \& MacGillivray, H. T. 1989, MNRAS, 238,379

Kaiser, N. 1986, MNRAS, 222, 323

Kerscher, M., Mecke, K., Schuecker, P., et al. 2001, A\&A, 377, 1

Kurtz, M. J., \& Mink, D. J. 1998, PASP, 110, 934

Ledlow, M. J., Loken, C., Burns, J. O., Owen, F. N., \& Voges, W. 1999, ApJ, 516, L53

MacGillivray, H. T., \& Stobie, R. S. 1984, Vistas Astr., 27, 433

Maddox, S. J., Efstathiou, G., Sutherland, W. J., \& Loveday, J. 1990, MNRAS, 243, 692

Olowin, R., De Souza, R. E., \& Chincarini, G. 1988, A\&AS, 73, 125

Ortiz-Gil, A., Guzzo, L., Schuecker, P., Böhringer, H., \& Collins, C. A. 2004, MNRAS, 348, 325
Pierpaoli, E., Borgani, S., Scott, D., et al. 2003, MNRAS, 342, 163 Pierre, M., Boehringer, H., Ebeling, H., et al. 1994, A\&A, 290, 725 Reiprich, T. H., \& Böhringer, H. 2002, ApJ, 567, 716

Romer, A. K., Collins, C. A., Böhringer, H., et al. 1994, Nature, 372, 75

Sánchez, A. G., Lambas, D. G., Bhringer, H., \& Schuecker, P. 2005, MNRAS, 362,1225

Sandage, A., Tammann, G. A., \& Yahil, A. 1979, ApJ, 232, 352

Schindler, S., Guzzo, L., Ebeling, H., et al. 1995, A\&A, 299, 9

Schindler, S., Hattori, M., Neumann, D. M., \& Boehringer, H. 1997, A\&A, 317, 646

Schuecker, P., Böhringer, H., Guzzo, L., et al. 2001, A\&A, 368, 86

Schuecker, P., Guzzo, L., Collins, C. A., \& Böhringer, H. 2002, MNRAS, 335 , 807

Schuecker, P., Böhringer, H., Collins, C. A., \& Guzzo, L. 2003a, A\&A, 398, 867 Schuecker, P., Caldwell, R. R., Böhringer, H., et al. 2003b, A\&A, 402, 53 Stanford, S. A., Romer, A. K., Sabirli, K., et al. 2006, ApJ, 646, 13 Stanek, R., Evrard, A. E., Böhringer, H., et al. 2006, ApJ, 648, 956 Struble, M. F., \& Rood, H. J. 1999, ApJS, 125, 35

Tonry, J. L., \& Davis, M. 1979, AJ, 84, 1511

Voges, W., Aschenbach, B., Boller, T., et al. 1999, A\&A, 349, 389 